\title{
Ingeniería Conducida por Modelos en Sistemas de Automatización Flexibles
}

\author{
R. Priego*, E. Estévez**, D. Orive*, I. Sarachaga*, M. Marcos* \\ *Dpto. Ingeniería de Sistemas y Automática, UPV/EHU, España \\ email: \{rafael.priego, dario.orive, sabel.sarachaga,marga.marcos\}@ehu.eus \\ **Dpto. Ingeniería Electrónica y Automática, EPS de Jaén, España \\ email: eestevez@ujaen.es
}

\begin{abstract}
Resumen
Los sistemas de automatización industrial actuales tienen que hacer frente a los desafios que surgen al tratar de solventar las necesidades generadas por un mercado altamente competitivo. Por ello, los sistemas de automatización modernos tratan de ofrecer soluciones más flexibles y eficientes con objeto de satisfacer requisitos como: eficiencia energética, optimización de rendimiento $y / o$ tolerancia a fallos en el proceso o controlador. El hecho de añadir mecanismos de reconfiguración en este tipo de sistemas permite alcanzar muchos de estos requisitos pero a cambio de incrementar la complejidad en diseño e implementación. Este trabajo explora las ventajas de la Ingeniería Conducida por Modelos para manejar dicha complejidad. En concreto, se presenta una plataforma basada en modelos que ayuda y guía en el diseño y desarrollo de sistemas de automatización flexibles. La aproximación propuesta está fundamentada en dos estándares muy aceptados en el sector de la automatización: AutomationML e IEC 61131-3.
\end{abstract}

Palabras Clave: Sistemas de Automatización Flexibles, Ingeniería Conducida por Modelos, AutomationML, IEC 61131-3, PLCopen.

\section{INTRODUCCIÓN}

Actualmente, instituciones públicas de países como Alemania [4], Estados Unidos [7] e incluso a nivel de Europa [16] están haciendo una apuesta en la automatización industrial con objeto de fomentar la innovación, empleo y crecimiento económico. Estas iniciativas persiguen la satisfacción de requisitos de integración, reutilización, flexibilidad así como la optimización del proceso de fabricación mediante soluciones de alta tecnología fundamentadas en equipos y sistemas adaptables e inteligentes [2].

En este contexto, los sistemas de automatización han comenzado a soportar mecanismos de reconfiguración dinámica con objeto de garantizar requisitos no funcionales entre los que se encuentran aquellas propiedades que hacen que los productos sean más atractivos, utilizables, fiables o seguros sin modificar la funcionalidad del producto.

El término de reconfiguración se puede utilizar para referirse o representar diferentes situaciones [18], [12], [24]. Este trabajo usa dicho término como la capacidad de reubicar las diferentes funcionalidades sobre el sistema de control distribuido con objeto de optimizar algún tipo de calidad de servicio. Por ejemplo: rendimiento del controlador [6], consumo de la batería [8] o la continuidad del servicio a pesar de fallo en el controlador o en la red [14], [19].

Sin embargo, la integración de los mecanismos de reconfiguración dentro de los sistemas de producción de automatización implica un aumento de su complejidad en términos de tamaño, funcionalidad y distribución, lo que hace que su diseño y desarrollo sean más complejos. En este contexto, se ha demostrado que el uso de la Ingeniería Conducida por Modelos (MDE- Model Driven Engineering) es adecuado para guiar y ayudar en las fases de diseño, desarrollo e implementación de sistemas complejos [17].

El uso de MDE en la automatización industrial no es nuevo [5], [9], [11], [20], [21], [23]. En los últimos años, se han publicado trabajos que hacen uso de estas técnicas para extender la caracterización de los sistemas (e.g. controladores, máquina, Unidades de programación POU-Program Organization Units- ...) para poder soportar el concepto de reconfiguración. [10] caracteriza la vista del software con información relacionada a las demandas de recursos (cantidad de memoria y número de bytes intercambiados con otros bloques de función FB) y esta información se utiliza para decidir los nodos del sistema en los que se desplieguen los FB. [22] introduce requisitos funcionales y no funcionales como restricción en las diferentes vistas de producción del sistema de automatización, comenzando por los sensores y actuadores hasta la planta completa. [3] presenta una caracterización de requisitos no funcionales basada en aspectos para sistemas de control embebidos.

El objetivo de este trabajo va un paso más allá de los trabajos comentados anteriormente. Se presenta una plataforma basada en modelos que permite generar 
los mecanismos necesarios para asegurar una determinada calidad de servicio a los sistemas de automatización. Se trata de una plataforma construida a partir de dos estándares bien aceptados en el sector que además tienen notación de Lenguaje de Marcado (ML-Markup Language), como son: AutomationML [1] y PLCopenXML [13]. Por lo tanto, las contribuciones de dicho trabajo son: (1) una aproximación de modelado que captura la información relevante del proceso de producción y el sistema distribuido de automatización, así como, cómo pasarlo a información valiosa para la plataforma de gestión en tiempo de ejecución; (2) un conjunto de herramientas que implementa la aproximación añadiendo flexibilidad a los sistemas de automatización originales, soportando una reconfiguración dinámica del sistema de control.

La estructura del trabajo es la siguiente: la sección 2 presenta una aproximación basada en AutomationML para modelar sistemas de automatización flexibles. Las secciones 3 y 4 se centran en la generación automática de código. Concretamente, la sección 3 detalla la generación de proyectos de automatización flexibles, mientras que la sección 4 detalla el procedimiento a seguir para la generación de código dependiente de la aplicación. La sección 5 presenta un caso de estudio y finalmente las conclusiones más relevantes se detallan en la sección 6 .

\section{DISEÑO DE UN SISTEMA DE AUTOMATIZACIÓN RECONFIGURABLE}

Esta sección en primer lugar define lo que se entiende por un sistema de automatización reconfigurable y posteriormente propone las pautas de su diseño haciendo uso de MDE y del estándar AutomationML.

\subsection{SISTEMA DE AUTOMATIZACIÓN RECONFIGURABLE}

Los módulos que forman un sistema de automatización reconfigurable son los siguientes:

- Controladores autómatas programables (PLCProgrammable Logic Controller) distribuidos.

- Componentes Mecatrónicos (MC- Mechatronic Components). Cada MC gestiona una parte del proceso (mecánica, eléctrica y electrónica) a través de instrumentación y hardware de control $y$ una pieza de software que puede ejecutarse en diferentes PLCs.

- Plataforma de ejecución, responsable de garantizar el cumplimiento total de la calidad de servicio del sistema en tiempo de ejecución.

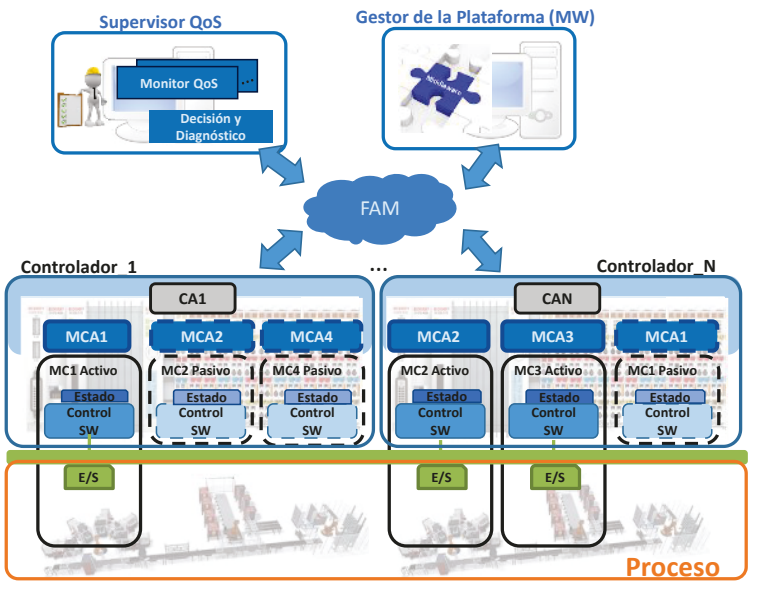

Agentes Dependientes de la aplicación: un CA por cada controlador (CA1..N) y un MCA por cada MC en el controlador

Fig. 1: Escenario general de un sistema de automatización reconfigurable

La Fig. 1 presenta el escenario general para los sistemas de automatización reconfigurables. Este trabajo adopta como plataforma de gestión FAM (Flexible Automation Middleware) que personaliza un MAS (Multi Agent System) genérico para monitorizar una determinada calidad de servicio (fallo de controlador, balance de carga...) lanzando una reconfiguración del sistema en caso de no estar cumpliéndose [15].

La reconfiguración implica desactivar componentes mecatrónicos en algunos controladores y activarlos en otros sin alterar la funcionalidad de la aplicación. Para poder activar y desactivar MCs, también se han de caracterizar por sus posibles modos de operación:

- Estado no crítico: aquellos estados donde el sistema de automatización sabe el estado actual del proceso, por lo que los MCs pueden ser activados en otro controlador con el estado inicial que sea el último estado conocido del MC desactivado.

- Estado crítico: aquellos estados donde el sistema de automatización no conoce exactamente el estado actual del MC. Los estados críticos inhiben la activación / desactivación de MCs para evitar comportamientos impredecibles del proceso. Por ejemplo después de fallo de controlador es necesario analizar si todos los MCs que se estaban ejecutando en él pueden ser recuperados en un estado previo conocido (checkpoint) o por el contrario no es posible y por lo tanto hay que parar al MC de forma segura y avisar al operario (non-recovery).

Finalmente es importante destacar que FAM también es responsable de asegurar que en ejecución todos los MCs de la aplicación estarán activos en un solo controlador. Para ello, incluye cuatro tipos de 
agentes. Dos de ellos forman parte de la arquitectura básica para el manejo de calidad de servicio: (1) Gestor de la plataforma (MW) y (2) Supervisor que a su vez está formado por un agente de monitorización y un agente Decisión y Diagnóstico. Otros dos dependen de la aplicación de automatización: (3) Agente de Controlador (CA-Controller Agent) para registrar el controlador y sus recursos y (4) Agente de MC (MCA-Mechatronic Component Agent) para manejar la ejecución del MC. Tal y como se resalta en Fig. 1 habrá un CA por controlador y tantos MCAs como MCs tenga vinculados dicho controlador.

\subsection{AML Y DISEÑO DEL SISTEMA}

Los desarrolladores diseñan e implementan los sistemas de automatización como un conjunto de MCs que utilizan un conjunto de E/S, POUs y variables. Además, cada $\mathrm{MC}$ se caracteriza por un conjunto de intervalos críticos definidos por una condición. Dichos MCs se replican en los diferentes controladores del sistema.

Para facilitar el diseño de este tipo de sistemas, este trabajo ha hecho uso de AutomationML (AML) al que le ha añadido los mecanimos necesarios para que los desarrolladores puedan especificar las características de los MCs. De hecho, se ha definido una librería CAEX teniendo en cuenta los conceptos que aparecen en los sistemas de automatización flexibles. La Fig. 2 detalla la librería CAEX definida. La Fig. 3 presenta un ejemplo de diseño de un sistema de automatización flexible.

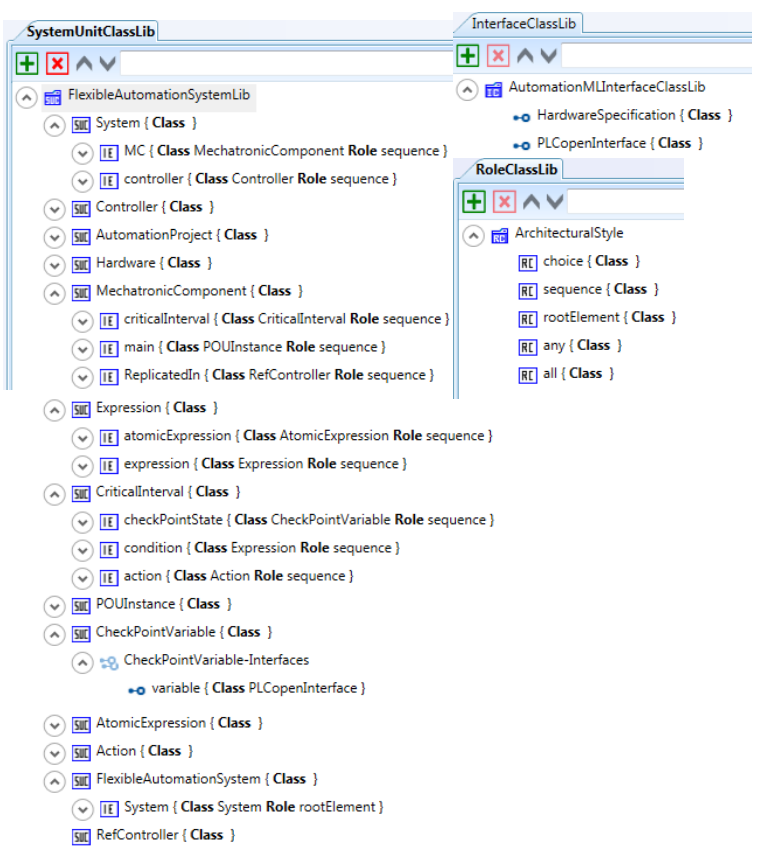

Fig. 2: Librería CAE para Sistemas de Automatización Flexibles

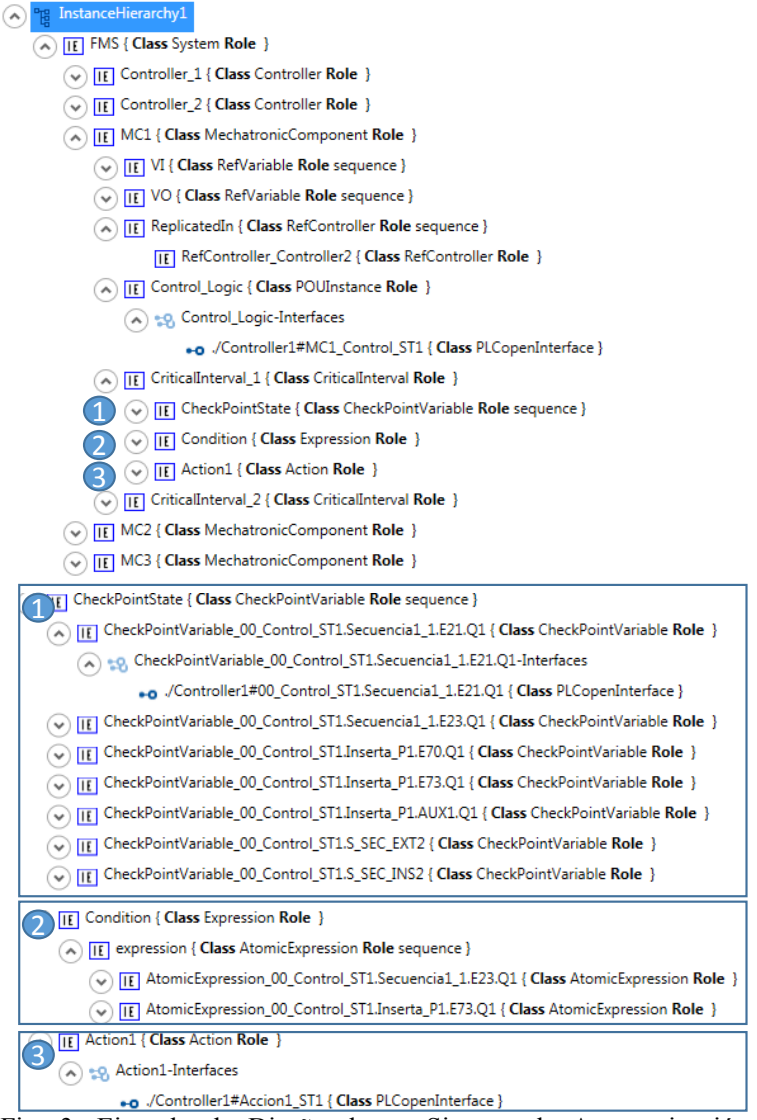

Fig. 3: Ejemplo de Diseño de un Sistema de Automatización Flexible

\section{GENERACIÓN AUTOMÁTICA DE UN PROYECTO DE AUTOMATIZACIÓN FLEXIBLE}

Actualmente los entornos de ejecución estándar IEC 61131-3 no soportan despliegue dinámico de código en tiempo de ejecución por lo que impiden una reconfiguración de la aplicación. Para solventar esta limitación, los proyectos de automación flexibles (generados automáticamente) contendrán la lógica de control de todos los MCs que se puedan ejecutar en el controlador. Por eso, este trabajo cuando se hace referencia a reconfiguración implica la desactivación de un MC en un controlador y la activación en otro. Otro aspecto a tener en cuenta es la gestión de ejecución de los MCs. Dicha gestión la realiza su correspondiente MCA para lo cual necesita consultar y actualizar el estado del MC. Por tanto, un proyecto de automatización flexible contiene no solo la funcionalidad de los MCs sino todo el código que les proporciona la flexibilidad. Dicha flexibilidad implica la generación automática de tres POUs:

- MCid_Control: programa de control que maneja la ejecución de la lógica del MC.

- MCid Serialize: programa que recoge y serializa el valor de las variables que forman el estado del MC. 
- MCid_Derialize: programa que de-serializa y fija los valores de las variables que forman el estado del MC.

El Generador automático de los Proyectos de Automatización Flexibles recoge información del modelo AML y haciendo uso de técnicas M2M genera el proyecto final en formato PLCopenXML.

Los siguientes sub-apartados detallan el procedimiento seguido.

\subsection{PROGRAMA QUE GESTIONA LA EJECUCIÓN DEL MC (MCid_Control)}

Este programa, que se generará de forma automática, permitirá al MCA correspondiente activar/desactivar la ejecución de la lógica así como lanzar las acciones de recuperación/parada del $\mathrm{MC}$ que gobierne. Como cualquier POU tiene dos partes bien diferenciadas: interfaz y cuerpo

La interfaz está compuesta por un conjunto de variables dependientes de la aplicación y tras locales estáticas que permiten al MCA gestionar la lógica de control de su MC. En concreto isActive y wasActive para gestionar la activación y desactivación del MC. Para dar soporte a la QoS de disponibilidad de controlador, hacen falta además recoveryAction $\mathrm{y}$ Action_CriticalIntervalID que permiten lanzar las acciones de recuperación cuando sea necesario antes de activar el MC.

Por otro lado la estructura del código es la siguiente:

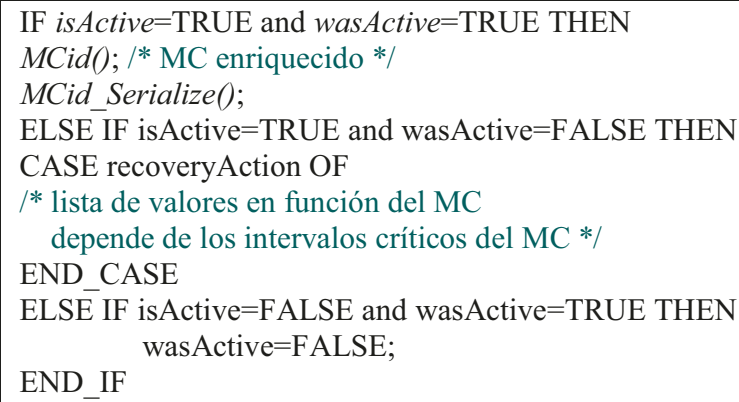

Finalmente, a través de una nueva regla de transformación se completa la lista de los posibles casos de activación del MC (isActive=TRUE and wasActive $=$ FALSE). La siguiente tabla detalla las plantillas de código en ST (Structured Text) a añadir en cada caso.

Tabla 1: Plantillas de código para estados no críticos y críticos
\begin{tabular}{|l|l|l|}
\hline $\begin{array}{c}\text { Direct } \\
\text { recovery }\end{array}$ & $\begin{array}{c}\text { Check Point } \\
\text { Recovery }\end{array}$ & \multicolumn{1}{c|}{ Safe Stop } \\
\hline & MCid_Action_id(); & \\
& IF(MCid_Action_id.end) & MCid_Action_id(); \\
MCid_Deserialize(); & THEN & IF(MCid_Action_id.end) \\
MCid (); & MCid_Deserialize(); & THEN \\
MCid_Serialize(); & recoveryAction=0; \\
wasActive=TRUE; & MCid_Serialize(); & isActive=FALSE; \\
& wasActive=TRUE; & END_IF \\
& recoveryAction=0; & \\
& END_IF & \\
\hline
\end{tabular}

\subsection{SERIALIZAR Y DE-SERIALIZAR EL ESTADO DEL MC}

El programa serializar es capaz de reunir los estados de ejecución en una matriz de bytes, para que sea accesible por el MCA. Así mismo, el programa deserializar sigue la misma estructura pero en lugar de recoger la información en un array, la extrae y reescribe el valor de las variables de estado.

Por ello, la interfaz de estos POUs será una variable local llamado state que será un array de bytes.

La funcionalidad ST del POU serializar es:

state[0]:=TypeOfGlobalVariable_TO_BYTE(GlobalVariable); ...

state[NumberOfBytes]:=TypeOfGlobalVariable_TO_BYTE(Glob alVariable);

Por otro lado la funcionalidad en ST del POU deserializar:

GlobalVariableName=BYTE_TO_TypeOfGlobalVariable (state[0]);

GlobalVariableName $=$ BYTE_TO_TypeOfGlobalVariable (state[NumberOfBytes]);

\section{GENERACIÓN AUTOMÁTICA DE AGENTES DE APLICACIÓN}

Para la generación de los agentes dependientes de aplicación se ha definido una plantilla (en Java) para los MCAs y otra para los CAs. Dichas plantillas tienen una serie de propiedades dependientes de la aplicación que se rellenan automáticamente por el generador de código que extrae información pertinente del fichero AML.

Los siguientes sub-apartados detallan la generación de MCAs y CAs respectivamente.

\subsection{AGENTES DE LOS COMPONENTES MECATRÓNICOS (MCA)}

Como se detalla en [15], los MCAs implementan una máquina de estados finitos (FMS), concretamente con los estados: Boot, Active, Tracking, Wait Decision y End. La Fig. 4 ilustra a través de un diagrama de clases UML la estructura general de la plantilla que ha sido implementada como una FSM de JADE.

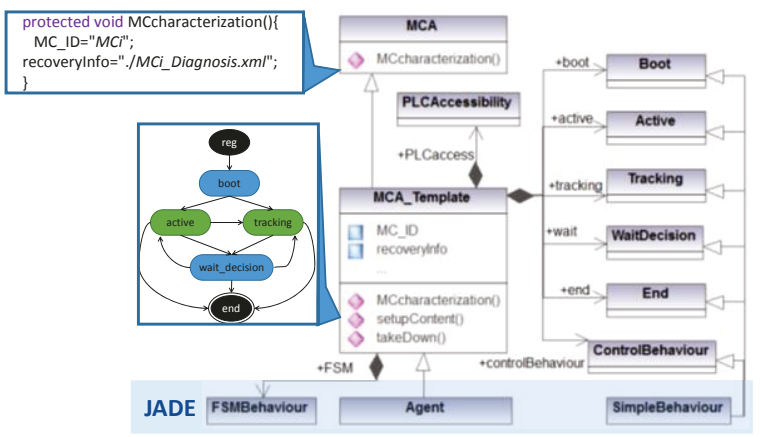

Fig. 4: Estructura general de MCA 
Cada estado tiene asociada una funcionalidad implementada como dos Behaviors de JADE (SimpleBehavior). Por otro lado, PLCAccesibility proporciona acceso al código de MC en el PLC.

Tiene dos propiedades parametrizables: $M C \_I D$ que es el ID del MC y recoveryInfo que direcciona al conjunto de máscaras que definen los intervalos críticos del MC. La siguiente figura presentan la estructura de dicho fichero a través de un XML Schema.

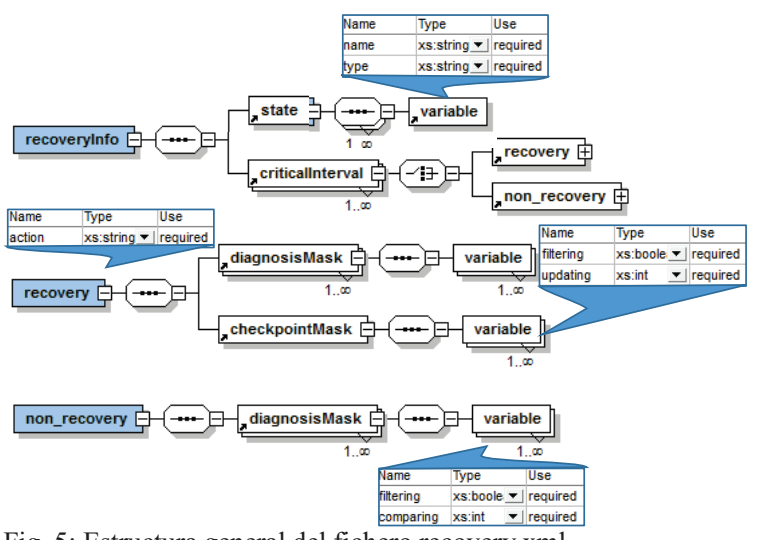

Fig. 5: Estructura general del fichero recovery.xml

Este fichero contiene información sobre las variables que forman el estado del MC, las máscaras para realizar el diagnóstico así como los checkpoint. Las máscaras de diagnóstico filtran las variables de estado relacionadas a la condición permitiendo así determinar el tipo de estado crítico (a checkpoint o irrecuperable).

\subsection{AGENTES DE CONTROLADOR (CA)}

Los CAs proporcionan información actualizada del estado de los recursos del controlador. Estos agentes también participan en el proceso de negociación cuando sea necesario. El criterio de negociación depende de la calidad de servicio que tenga asociada.

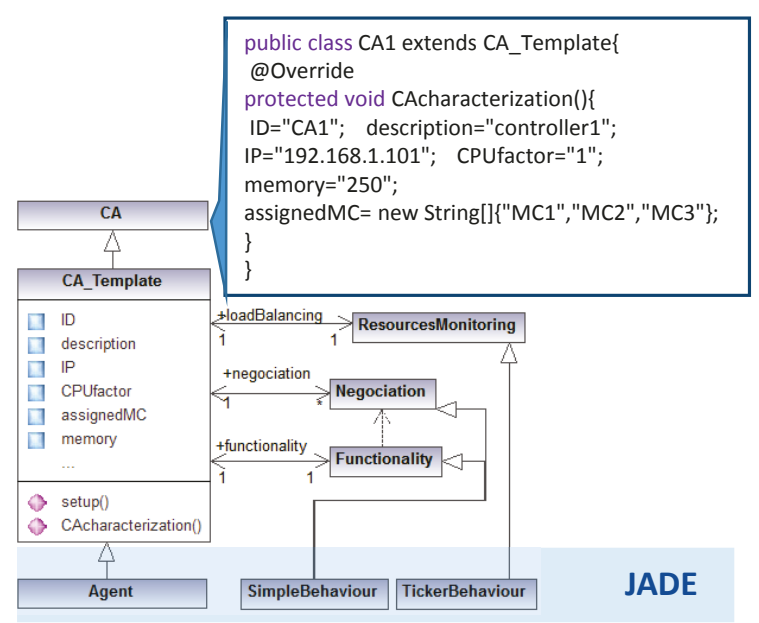

Fig. 6: Estructura general de la plantilla CA
La Fig. 6 detalla a través de un diagrama de clases UML la estructura general de la plantilla CA. La funcionalidad básica de CA se implemente en un "Behavior" cíclico que se gestiona los mensajes de los agentes de la plataforma. Por otro lado, el CA también implementa una monitorización de recursos del controlador. La plantilla definida tiene una serie de parámetros dependientes de la aplicación: ID, una descripción y un factor de CPU para tomar como referencia, recursos de memoria y la lista de MCs cuya lógica de control está vinculada al controlador (AssignedMC).

\section{CASO DE ESTUDIO}

La aproximación para el modelado y generación automática de proyectos de automatización flexibles propuesta en el presente trabajo ha sido aplicada con objeto de asegurar un balance de carga entre los controladores en un sistema de automatización como es la célula de fabricación flexible FMS-200. Esta célula consta de cuatro estaciones y un sistema de transporte que se encarga del ensamblado de 4 piezas (base, rodamiento, eje y tapa).

La primera estación se comprueba si la base está en la orientación correcta y si es así se coloca en el pallet situado en el sistema de transporte. En la segunda, se coloca el rodamiento y eje, mientras que la tapa se coloca en la tercera estación. Finalmente, la pieza montada se almacena en un almacén.

La célula está formada por 5 componentes mecatrónicos, uno por cada estación y otro para el sistema de transporte. Sin embargo, con objeto de simplificar la problemática únicamente se considerarán los MCs correspondientes a las tres primeras estaciones.

En lo referente al demostrador, éste está formado por dos controladores Beckhoff CX1020 donde se ejecutará la lógica de los MCs. Además estará dotado de un PC supervisor donde se ejecutarán el MW y el supervisor. La estación 1 tiene cinco intervalos críticos con sus correspondientes acciones que permiten que la estación continúe extrayendo las bases del almacén.

La Fig. 3 presenta parte de la especificación de los MCs indicados en el modelo AML, concretamente se resalta la caracterización del $\mathrm{MC} 1$.

Una vez aplicada las transformaciones indicadas en el trabajo se genera el proyecto de automatización flexible, los CAs y los MCAs junto con los ficheros de diagnóstico y se despliegan en los correspondientes controladores.

Por ejemplo, la siguiente figura ilustra parte del proyecto de automatización flexible generado para el controlador 1 en formato PLCopen XML. En rosa aparece remarcado los POUs procedentes de proyecto de automatización modular y en verde los POUs generados automáticamente. 


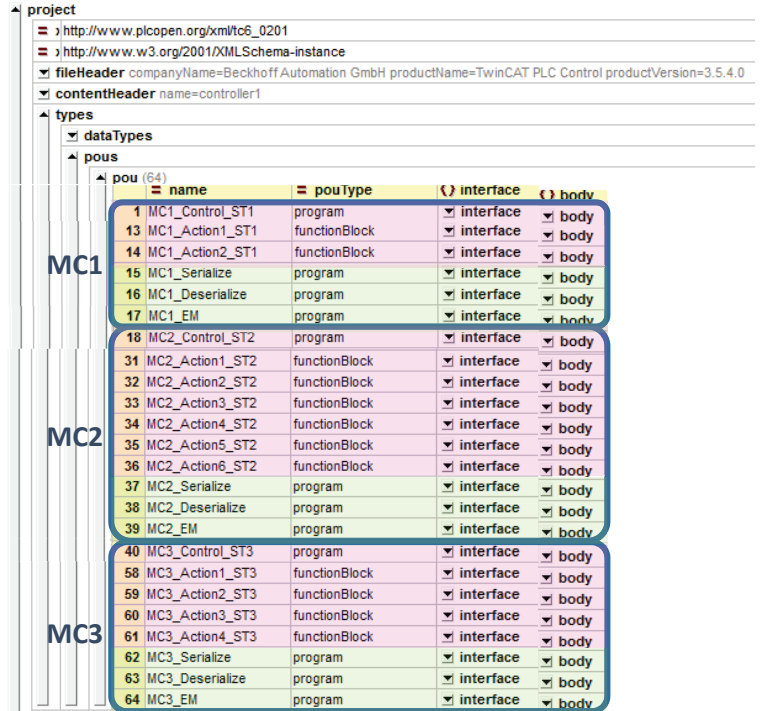

Fig. 7: Proyecto de Automatización Flexible para el controlador 1 From the Modular Automation Project

Se Abramaticaligadereduna serie de pruebas de reconfiguración en tiempo de ejecución. En concreto, se ha comenzado teniendo arrancado únicamente el controlador 1 como si se tratase de una aplicación centralizada. Posteriormente se ha arrancado el controlador 2. Después de registrarse, la monitorización de la carga detecta que dicho controlador está muy por debajo de la carga especificada. Así el CA2 envía un evento de balance de carga a FAM quien decide una nueva distribución basándose en los factores de CPU de los CAs, distribución actual de los MCs, así como la máxima carga de CPU que introducen los MCs. Una vez obtenida la nueva distribución empieza el proceso de recolocación. Durante este proceso, el MCA activo para la ejecución del código de control en un estado no crítico. Después del proceso de reconfiguración, MC1 y MC2 se ejecutan en el controlador 1 y MC3 en el controlador 2. La Fig.8 presenta a modo de gráfica la carga de CPU de los controladores antes y después de la reconfiguración.

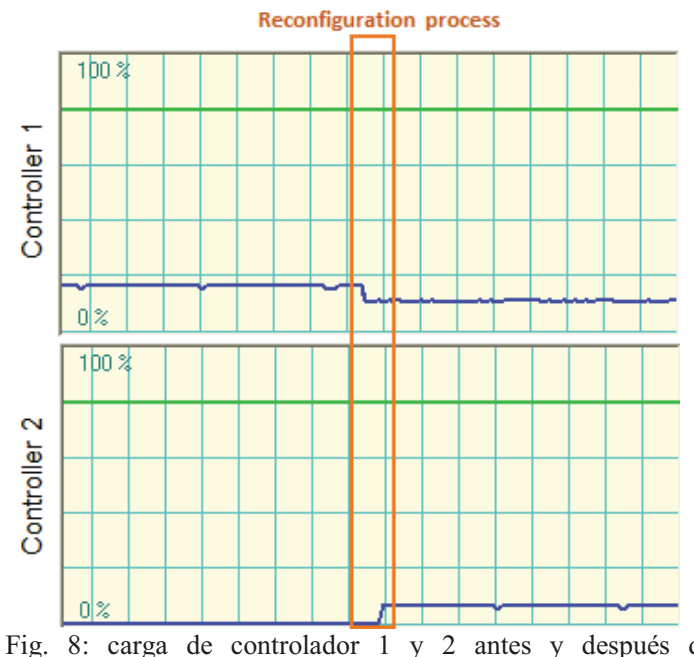

Fig. 8: carga de controlador 1 y 2 antes y después de la reconfiguración

\section{CONCLUSIONES}

Este trabajo ha presentado una aproximación centrada en añadir flexibilidad a los sistemas de automatización, permitiendo así soportar reconfiguración de los sistemas de control.

Se ha hecho uso de una plataforma de gestión basada en MAS que proporciona gestión de QoS en tiempo de ejecución. Los autores, proponen un diseño basado en modelos que permite capturar la información relevante sobre los procesos de producción, sistemas de automatización distribuidos, así como, cómo hacer uso de dicha información para gestionar la plataforma. Así la solución propuesta proporciona soporte al ciclo desarrollo donde el código resultante es: un proyecto de automatización flexible por cada controlador, tantos CAs como controladores hayan en el sistema y por cada controlador tantos MCAs y MCs como él maneje.

Por lo tanto, la solución propuesta permite añadir flexibilidad a los proyectos de automatización modulares originales, soportando reconfiguración dinámica del sistema de control.

\section{Agradecimientos}

Este trabajo ha sido financiado por el proyecto DPI2015-68602-R (MINECO/FEDER, UE), por la UPV/EHU en el marco del proyecto PPG17/56 y GV/EJ en el marco de grupos de investigación reconocidos IT914-16.

\section{Referencias (10 ptos, negrita)}

[1] AutomationML (2017) http://www.automationml.org

[website]

[2] Association EF of the FR. Factories of the Future PPP FoF 2020 Roadmap: Consultation document. 2012.

[3] Binotto APD, Wehrmeister MA, Kuijper A, Pereira CE. Sm@rtConfig: A context-aware runtime and tuning system using an aspectoriented approach for data intensive engineering applications. Control Eng Pract 2013;21:204-17. doi:10.1016/j.conengprac.2012.10.001.

[4] Blanchet M, Rinn T, Von Thaden G, de Thieulloy G. Industry 4.0 The new industrial revolution How Europe will succeed. 2014.

[5] Booch G, Rumbaugh J, Jacobson I. The Unified Modeling Language User Guide (2nd Edition). Addison-Wesley Professional; 2015.

[6] Botygin IA, Tartakovsky VA. The Development and Simulation Research of Load Balancing Algorithm in Network Infrastructures. Int. Conf. Mech. Eng. Autom. Control Syst., 2014, p. 0-4. doi:10.1109/MEACS.2014.6986904.

[7] European Commission: Research and Innovation. Factories of the Future PPP: towards competitive EU manufacturing. 2013. 
[8] Guo L, Wang B, Wang W. Research of energyefficiency algorithm based on on-demand load balancing for wireless sensor networks. Proc Int Symp Test Meas 2009;2:22-6. doi:10.1109/ICTM.2009.5413071.

[9] Estevez E, Marcos M, Gangoiti U, Orive D. A Tool Integration Framework for Industrial Distributed Control Systems. Proc. 44th IEEE Conf. Decis. Control, IEEE; 2005, p. 8373-8. doi:10.1109/CDC.2005.1583518.

[10]Fay A, Vogel-Heuser B, Frank T, Eckert K, Hadlich T, Diedrich C. Enhancing a modelbased engineering approach for distributed manufacturing automation systems with characteristics and design patterns. J Syst Softw 2015;101:221-35. doi:10.1016/j.jss.2014.12.028.

[11] Hästbacka D, Vepsäläinen T, Kuikka S. Modeldriven development of industrial process control applications. J Syst Softw 2011;84:1100-13.

[12] Krupitzer C, Roth FM, VanSyckel S, Schiele G, Becker C. A survey on engineering approaches for self-adaptive systems. Pervasive Mob Comput 2014;17:184-206. doi:10.1016/j.pmcj.2014.09.009.

[13] Marcos M, Estevez E, Perez F, Van der Wal E. XML exchange of control programs. IEEE Ind Electron Mag 2009;3:32-5. doi:10.1109/MIE.2009.934794.

[14] Merz M, Frank T, Vogel-Heuser B. Dynamic redeployment of control software in distributed industrial automation systems during runtime. 2012 IEEE Int. Conf. Autom. Sci. Eng., IEEE; 2012, p. 863-8. doi:10.1109/CoASE.2012.6386445.

[15] Priego R, Iriondo N, Gangoiti U, Marcos M. Agent Based Middleware Architecture for Reconfigurable Manufacturing Systems. Int J Adv Manuf Technol (2017). doi:10.1007/s00170-017-0154-z. In Press.

[16] Science N, Council T. ADVANCED MANUFACTURING: A Snapshot of Priority Technology Areas Across the Federal Government Subcommittee for Advanced Manufacturing 2016.

[17] Selic B. The pragmatics of model-driven development. IEEE Softw 2003;20:19-25. doi:10.1109/MS.2003.1231146.

[18] Shen W, Wang L, Hao Q. Agent-based distributed manufacturing process planning and scheduling: a state-of-the-art survey. IEEE Trans Syst Man Cybern Part C (Applications Rev 2006;36:563-77. doi:10.1109/TSMCC.2006.874022.

[19] Streit A， Rösch S, Vogel-Heuser B. Redeployment of Control Software during Runtime for Modular Automation Systems Taking Real-Time and Distributed I/O into Consideration. IEEE 19th Conf. Emerg.
Technol. Fact. Autom. (ETFA), 2014, 2014, p. 1-4.

[20] SysML. The SysML Specification, v 1.0 2007. http://www.sysml.org

[21] Thramboulidis K, Perdikis D, Kantas S. Model driven development of distributed control applications. Int $\mathrm{J}$ Adv Manuf Technol 2006;33:233-42. doi:10.1007/s00170-006-04550 .

[22] Vogel-Heuser B, Rösch S. Integrated Modeling of Complex Production Automation Systems to Increase Dependability. Risk - A Multidiscip. Introd., 2014, p. 1-476. doi:10.1007/978-3-31904486-6.

[23] Vyatkin V, Hanisch H-M. Closed-Loop Modeling in Future Automation System Engineering and Validation. IEEE Trans Syst Man, Cybern Part C (Applications Rev 2009;Vol: 39 pp:17-28. doi:10.1109/TSMCC.2008.2005785.

[24] ang L, Adamson G, Holm M, Moore P. A review of function blocks for process planning and control of manufacturing equipment. J Manuf Syst 2012;31:269-79. doi:10.1016/j.jmsy.2012.02.004. 